In the 1970s, Freeman Dyson lamented what he saw as the high degree of alienation between theoretical physicists and mathematicians. After 1980, however, it was internationally recognized that a renaissance had begun in the exchange of knowledge between pure mathematics and theoretical physics. Edward Witten has played a remarkable role in sparking that renaissance.

\section{Biographical Sketch}

Edward Witten was born in Baltimore, Maryland, in 1951. He received his Ph.D. in physics from Princeton University in 1976. Between 1976 and 1980 he was first a postdoctoral fellow and then a junior fellow of the Society of Fellows at Harvard University. In 1980 he became professor at Princeton University. He has been a professor at the Institute for Advanced Study since 1987 and has been Charles Simonyi Professor there since 1997.

He received the Dirac Medal of the Abdus Salam International Centre for Theoretical Physics in 1985 and was awarded the Fields Medal in 1990. He received the Dannie Heineman Prize of the American Physical Society in 1998, the Crafoord Prize of the Royal Swedish Academy of Sciences in 2008, and the Lorentz Medal of the Royal Netherlands Academy of Arts and Sciences in 2010. In 2002 he was awarded the National Medal of Science, and in 2012 he received the Breakthrough Prize in Fundamental Physics. He is a member of the American
Academy of Arts and Sciences, the National Academy of Sciences, and the Royal Society.

\section{About the Prize}

The Kyoto Prize is Japan's highest private award for global achievement, honoring significant contributions to the betterment of society. The Inamori Foundation is a nonprofit organization established in 1984 by Kazuo Inamori, founder and chairman emeritus of Kyocera and KDDI Corporation. The activities of the Inamori Foundation reflect the lifelong belief of its founder that a human being has no higher calling than to strive for the greater good of society and that the future of humanity can be assured only when there is a balance between scientific progress and spiritual depth. The Kyoto Prize is presented not only in recognition of outstanding achievements but also in honor of the excellent personal characteristics that have shaped those achievements.

Previous Kyoto Prize winners who have made contributions to the mathematical sciences are: Rudolf E. Kalman (1985), Claude E. Shannon (1985), John McCarthy (1988), I. M. Gelfand (1989), Edward Lorenz (1991), André Weil (1994), Donald E. Knuth (1996), Kyosi Itô (1998), Mikhael Gromov (2002), Simon A. Levin (2005), Hirotugu Akaike (2006), and László Lovász (2010).

- From Inamori Foundation announcements

\title{
2014 Nevanlinna Prize Awarded
}

On August 13, 2014, the 2014 Rolf Nevanlinna Prize was awarded at the opening ceremonies of the International Congress of Mathematicians (ICM) in Seoul, Korea. The prizewinner was SuBHASH KHOT of the Courant Institute of Sciences, New York University.

In 1982 the University of Helsinki granted funds to award the Nevanlinna Prize, which honors the work of a young mathematician (less than forty years of age) in the mathematical aspects of information science. The prize is presented every four years by the International Mathematical Union (IMU). Previous recipients of the Nevanlinna Prize are: Robert Tarjan (1982), Leslie Valiant (1986), Alexander Razborov (1990), Avi Wigderson (1994),

DOI: http://dx.doi.org/10.1090/noti1182
Peter Shor (1998), Madhu Sudan (2002), Jon Kleinberg (2006), and Daniel Spielman (2010).

Khot was honored "for his prescient definition of the 'Unique Games' problem and leading the effort to understand its complexity and its pivotal role in the study of efficient approximation of optimization problems; his work has led to breakthroughs in algorithmic design and approximation hardness, and to new exciting interactions between computational complexity, analysis and geometry."

Typically, major math prizes are given for major results. But in this case, Subhash Khot received the Nevanlinna Prize in large part for a conjecture-and even more surprisingly, one whose truth experts can't yet decide on.

But Khot's Unique Games Conjecture has already amply proven its value, even should it ulti- 
mately be disproven. It has cast a bright light on previously dim areas of computational complexity and provided critical insight-and, yes, Khot has also used it to prove major results, ones that stand regardless of its truth.

The conjecture has opened up a particularly fruitful way of addressing the central question of the field of computational complexity: How hard are problems to solve? More precisely, if you found the cleverest possible way to solve a particular problem, how quickly could a computer find the answer using it?

Computer scientists are nearly certain that some problems are so difficult that computers can't reliably find the answer at all, at least not in any reasonable amount of time (such as before the universe ends). That's the famous conjecture known as $P \neq N P$, and it has resisted proof for four decades-though computer scientists have only become more convinced that it must be true over time.

So many researchers have moved on to the next question: If a problem is too hard for a computer to solve quickly and precisely, can it at least find a good approximation? In the real world, after all, a good approximation is usually enough.

Before Khot's work, researchers had found a few problems for which the answer was no, but for most problems, they had little idea. Khot found a remarkably simple problem (called Unique Games) that seems to encapsulate what makes many problems hard to solve even approximately in a reasonable amount of time. His conjecture is that it's not just hard but impossible to reliably find an approximate answer to Unique Games reasonably quickly. In a certain precise, technical sense, Unique Games seems to be the simplest really, really hard problem.

Whether he's right or wrong, his problem has "cleaved nature at its joints," as the early taxonomist Carl Linnaeus put it. The conjecture is proving to be a kind of lever point, a spot where applying effort yields big results. Assuming the conjecture is true, Khot and others have shown that the vast majority of problems computer scientists care about also can't be approximated. Not only that, but the conjecture has shed light on seemingly unrelated problems in geometry, Fourier analysis, and even the mathematics of foams and voting, and those results don't rely on its truth.

The Unique Games problem is an elaboration of one that a six-year-old could play with. Imagine that you have a box of crayons and a drawing of a bunch of bubbles, some of which have lines connecting them. (Computer scientists call such drawings "networks.") Can you find an efficient way to color in the bubbles so that any two connected ones are different colors?

If your box has only two crayons (say yellow and purple), you can figure this out quite efficiently.
Start with an arbitrary bubble and color it yellow. Since all the bubbles connected to it now have to be purple, color them in. Continue this way until you've either managed to color in the whole network or you've found a bubble that's connected to both yellow and purple bubbles, making the project impossible.

If you add just one more crayon, though, this method fails, because when you color the first bubble yellow, you don't know what color the connected ones have to be. So if you get to a bubble you can't color in without breaking the rules, you don't know if a different selection earlier would have solved the problem. The difficulty isn't just with that method; no other

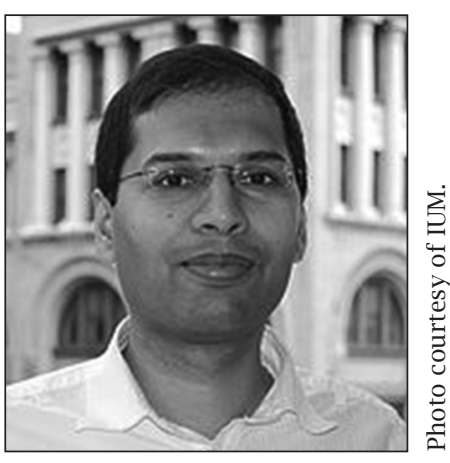

Subhash Khot method will reliably and efficiently solve the problem either. It has been proven to be NP-hard-in other words, effectively impossible.

Khot altered this problem slightly. He made it easier than the ordinary three-crayon problem by providing a rule so that whenever any bubble is colored in, the color of all connected bubbles is fixed. Then an algorithm like the earlier one applies, and it's easy enough to determine if the network can be colored in without breaking the rules. But for networks that are duds-ones that can't be colored in-Khot asked this: Which coloring breaks the fewest rules possible?

The Unique Games Conjecture (UGC) is that if you have lots of colors, you'll never find an efficient method to color in the drawing that's anywhere close to the best one, no matter how clever you are.

Khot developed the conjecture in 2001 (in a slightly different formulation, from which the name was derived). A couple of years later, computer scientists got the first glimmer of its importance, when Khot and others found that if the UGC was true, then they could find firm limits on how well many other problems could be approximated.

Here's one: Imagine at a celebrity-studded party guests sought out the stars to shake hands with them (but noncelebrity partygoers kept their hands to themselves with one another). If you know only who shook hands with whom, can you figure out the minimum number of celebrities that might have been at the party? A simple algorithm can find an approximate solution for this, but it might specify as many as twice as many celebrities as are really needed. Computer scientists long imagined you could do better with a more sophisticated algorithm, but in 2003, Khot, together with Oded Regev, showed that if the UGC is true, they were wrong. That simple algorithm is the best you can do. 
Khot's next success came in 2005. Together with Ryan O'Donnell, Elchanan Mossel, and Guy Kindler, Khot found that the UGC implied a similar limit for approximating solutions to a problem called "Max Cut," which asks you to take a network and split it into two groups so that the maximum number of edges passes between them. Then in 2008, Prasad Raghavendra showed that if the UGC is true, a very simple method can find the best approximations for an enormous class of problems called "constraint satisfaction problems." With this, computer scientists know exactly how well nearly any problem can be approximated.

Of course, the caveat is that all these results depend on the UGC being true. If it turns out to be false, the entire sparkling, beautiful theory is a mirage. But the conjecture has proved remarkably powerful independent of its truth. In the process of using the UGC to discover how well other problems could be approximated, Khot and others have proven several significant theorems in other areas, including geometry and Fourier analysis.

These implications have even ranged as far as voting theory. Once all the votes in an election are cast, there are a variety of ways of determining the winner. One of the most obvious is that the election goes to the candidate with the majority of votes, but there are other choices too, such as the American Electoral College system. Khot and his coauthors used intuition from the UGC to propose that majority-rules is the method for counting votes in which a few miscounted votes is least likely to change the election result. This was indeed confirmed later by others.

Another group was working on proving that the UGC was true, and while their method failed, the work led them to another discovery: They found a shape that in a certain sense lies halfway between a square and a circle (though in many more than two dimensions).

Like a square, copies can be placed next to each other horizontally and vertically to fill a whole space without gaps or overlaps, forming a multi-dimensional foam. But its perimeter is much smaller than a square-it's closer to that of a circle, the object which has the smallest perimeter for the area contained.

In the meantime, other researchers have worked to prove that the UGC is wrong, with equal lack of direct success but with equal collateral benefits. Although they haven't yet succeeded in finding an algorithm that can efficiently find a good approximate solution to Unique Games, they have developed some excellent new algorithmic methods for other circumstances.

Efforts to prove the conjecture, to disprove it, and to discover its consequences have all proven enormously fruitful. The Unique Games Conjecture will be driving research in theoretical computer science for many years to come.
Subhash Khot was born in Ichalkaranji, India, in 1978. He received his Ph.D. in 2003 from Princeton University. He spent the year 2003-04 as a member of the School of Mathematics at the Institute for Advanced Study at Princeton. He was assistant professor at the College of Computing at the Georgia Institute of Technology from 2004 to 2007 and served as associate professor at New York University from 2007 to 2011. Between 2011 and 2013 he held a visiting position at the University of Chicago. He is currently a professor in the computer sciences department at the Courant Institute. He received the Microsoft Research New Faculty Fellowships Award in 2005 and the Alan T. Waterman Award from the National Science Foundation (NSF) in 2010; he also gave an invited talk at the ICM that year.

- From an IMU news release 